\title{
Sinochoanal Polyp of Sphenoidal Origin: Diagnostic and Surgical Approach in Two Cases of Large Sphenochoanal Polyp
}

\author{
${ }^{1}$ Boon C Gan, ${ }^{2}$ Ramiza R Ramli
}

\begin{abstract}
The diagnosis of sinochoanal polyp (SP) with the origin of either maxillary or ethmoidal sinuses may not be uncommon for any otorhinolaryngologist. We report two rare cases of large sphenochoanal polyp (SCP) that underwent complete excision using endoscopic transnasal approach, debulking with microdebrider, and subsequent evacuation of polyp through the oral cavity under image-guided surgery (IGS) protocol. Due to the rarity of SCP, this condition may sometimes be mistaken for antrochoanal polyp (ACP) and lead to unwarranted exploration of other sinuses and incomplete removal of SCP from the sphenoid sinus. In this report, we also discuss on the diagnostic confusion with ACP, the surgical approach, as well as the usage of endoscopic and imaging techniques in managing SCP.
\end{abstract}

Keywords: Antrochoanal polyp, Image-guided surgery, Nasal polyp, Natural orifice endoscopic surgery, Sinochoanal polyp, Sphenoid sinus.

How to cite this article: Gan BC, Ramli RR. Sinochoanal Polyp of Sphenoidal Origin: Diagnostic and Surgical Approach in Two Cases of Large Sphenochoanal Polyp. Int J Otorhinolaryngol Clin 2017;9(2):68-71.

Source of support: Nil

Conflict of interest: None

\section{INTRODUCTION}

Nasal polyposis is a commonly seen disease in the otorhinolaryngology clinic. However, SP constitutes only about 3 to $6 \%$ of nasal polyps. ${ }^{1}$ The SP can be classified into ACP, ethmochonal polyp, or SCP based on the pedicle's origin. ${ }^{1,2}$ Every otorhinolaryngologist will come across an ACP at some point of their career, but not all will be exposed to an isolated SCP due to its rarity. ${ }^{3}$ Owing to its unspecific presentation coupled with a similar appearance to ACP, the diagnosis may be difficult and confusing. ${ }^{3}$ The diagnosis of SCP is achieved based on the clinical features, endoscopic findings, and

\footnotetext{
${ }^{1}$ Senior Resident, ${ }^{2}$ Senior Consultant

1,2Department of Otorhinolaryngology-Head \& Neck Surgery Universiti Sains Malaysia, Kota Bharu, Kelantan, Malaysia

Corresponding Author: Boon C Gan, Senior Resident Department of Otorhinolaryngology-Head \& Neck Surgery Universiti Sains Malaysia, Kota Bharu, Kelantan, Malaysia Phone: +60105665008, e-mail: gbchye@gmail.com
}

imaging techniques. ${ }^{3}$ In recent years, with the emergence of technological advancements in endoscopy, computed tomography (CT), and magnetic resonance imaging (MRI), the previously underreported SCP is no longer as challenging to diagnose as before. ${ }^{4}$ The SCP is best treated surgically via complete excision to minimize recurrence. ${ }^{1,2,5}$ The purpose of this article is to share our experience in dealing with two cases of large SCP that underwent endoscopic sinus surgery (ESS) and focus on the aspect of diagnosis and management.

\section{CASE REPORTS}

\section{Case 1}

A 17-year-old female student with no previous medical illness presented to the clinic with a complaint of gradually worsening left nasal blockage for the past 5 years. The initial unilateral complaint slowly became persistent and subsequently progressed to bilateral nasal blockage with other complaints, such as hyposmia, headache, and facial discomfort. She also had mouth breathing, hyponasal voice, snoring, and daytime somnolence. Rigid nasal endoscopic examination showed hypertrophic and palelooking bilateral inferior turbinates with white glistening mass occupying the left nasal cavity and also part of the right choanal region (Fig. 1). There was copious inspissated mucoid discharge on the left nasal floor. However, the origin of the left nasal mass cannot be visualized and determined due to limited space for scope manipulation. The CT scan of the paranasal sinuses showed a large $3.0 \times$ $3.8 \times 5.8 \mathrm{~cm}$ mass that occupied the nasopharynx with extension into posterior choana and inferior extension to the soft palate (Fig. 2). There was complete opacification of left maxillary and left sphenoid sinus with mucosal thickening over the opposite maxillary and sphenoid sinus. Preoperative diagnosis of left ACP was made due to the patient's age, unilaterality of polyp, and symptoms that were more suggestive of infection in etiology. The patient was then scheduled for left ESS with IGS protocol under general anesthesia. After uncinectomy, uncapping of bulla ethmoidalis, and debulking of left nasal mass with microdebrider, we noted that the polyp occupying the posterior choana and nasopharynx actually originated from the left sphenoid sinus. The polyp was detached 

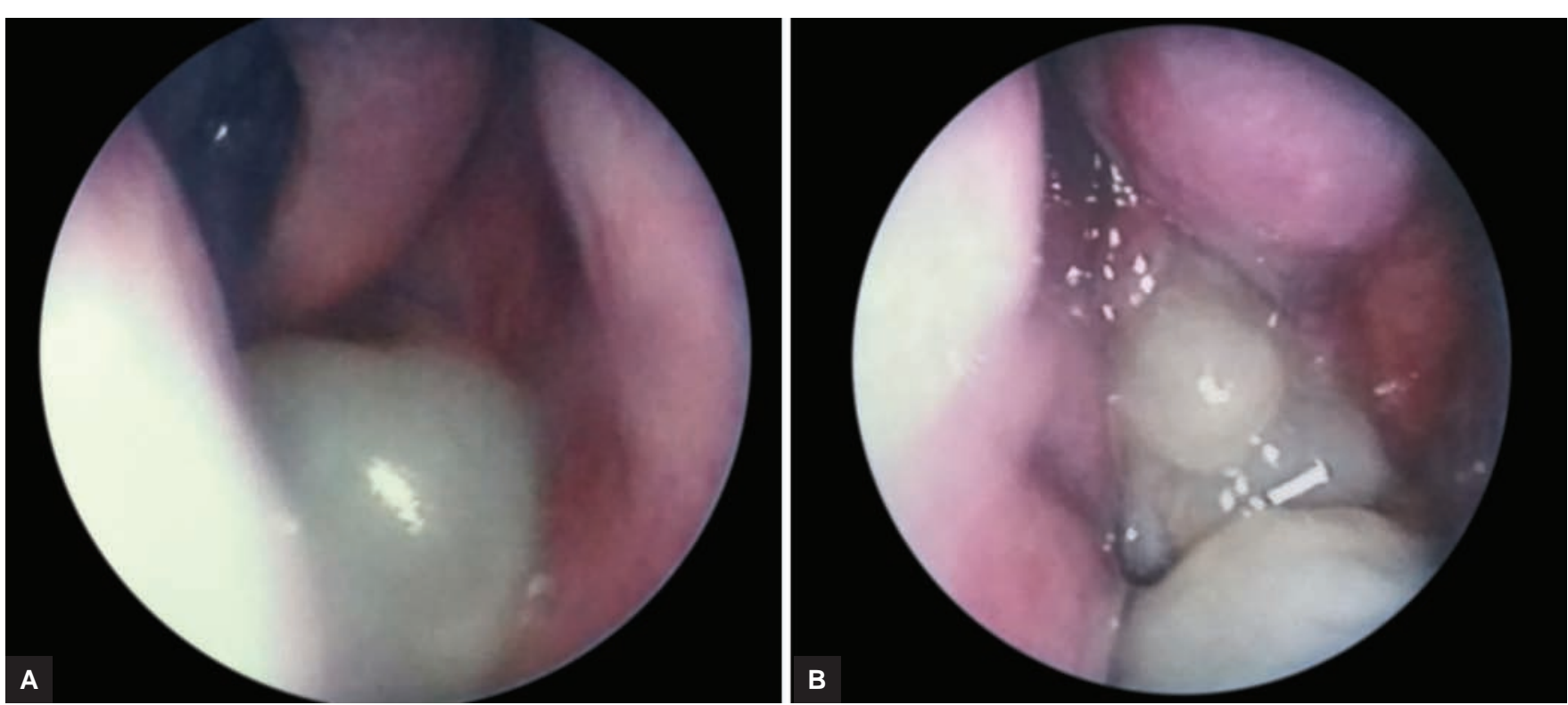

Figs 1A and B: Typical character of nasal polyp which appears as whitish, glistening mass upon examination using a rigid nasoendoscopy
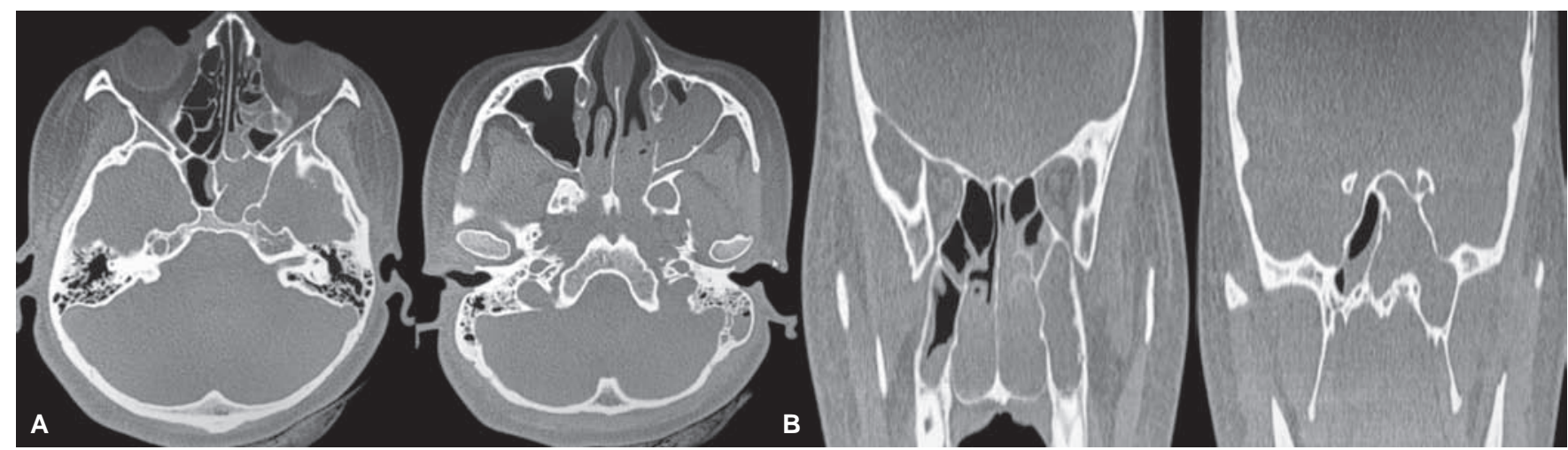

Figs 2A and B: Computed tomography scans taken from case report 1. There were complete opacification of the left maxillary and left sphenoid sinuses making the localization of sinochoanal polyp difficult, thus needing endoscopic sinus surgery as both diagnostic and therapeutic procedures

using cutting forceps, pushed down to the oropharynx, and removed via oral cavity. The left sphenoid ostium was widened and the remaining polyp in the sphenoid sinus was cleared with punch forceps. Histopathological examination confirmed the nasal mass as an inflammatory polyp. Postoperatively, her symptoms resolved and no sign of polyp was seen during the subsequent followup 3 months later.

\section{Case 2}

A 19-year-old healthy female had complaints of alternating nasal blockage for the past 3 years, predominantly on the right side. She also complained of occasional headaches, bilateral ears fullness, and had loud snoring without apnea spells. On examination, there was complete absence of cold spatula misting over the right side. Rigid nasoendoscopy showed bilateral pale, hypertrophic inferior turbinates with a white, glistening polypoidal mass that may be arising from the right osteomeatal complex. The CT scan of the paranasal sinuses revealed a large soft tissue lesion in the right nasal cavity, extending posterosuperiorly to the choana and nasopharynx (Fig. 3). She underwent right ESS under general anesthesia. Intraoperatively, after debulking of the polypoidal mass using microdebrider, we noted a large polyp, measuring $3.2 \times 3.0 \mathrm{~cm}$, arising from the right sphenoid sinus with minimal pus discharge. The polyp's stalk had already widened the sphenoid ostium and its attachment was identified (Fig. 4), and then separated using forceps before removal via the oral cavity. The specimen was confirmed to be inflammatory polyp by the pathologist (Fig. 5). On clinic follow-up 3 months later, the patient remained symptom-free with no signs of recurrence.

\section{DISCUSSION}

Solitary SCP with extension into the nasopharynx and pedicles attached to either the anterior or inferior wall of sphenoid sinus is a very rare discovery. ${ }^{6}$ Due to the scarcity, SCP may be mixed up with ACP, especially by budding otorhinolaryngologists because of their similar 


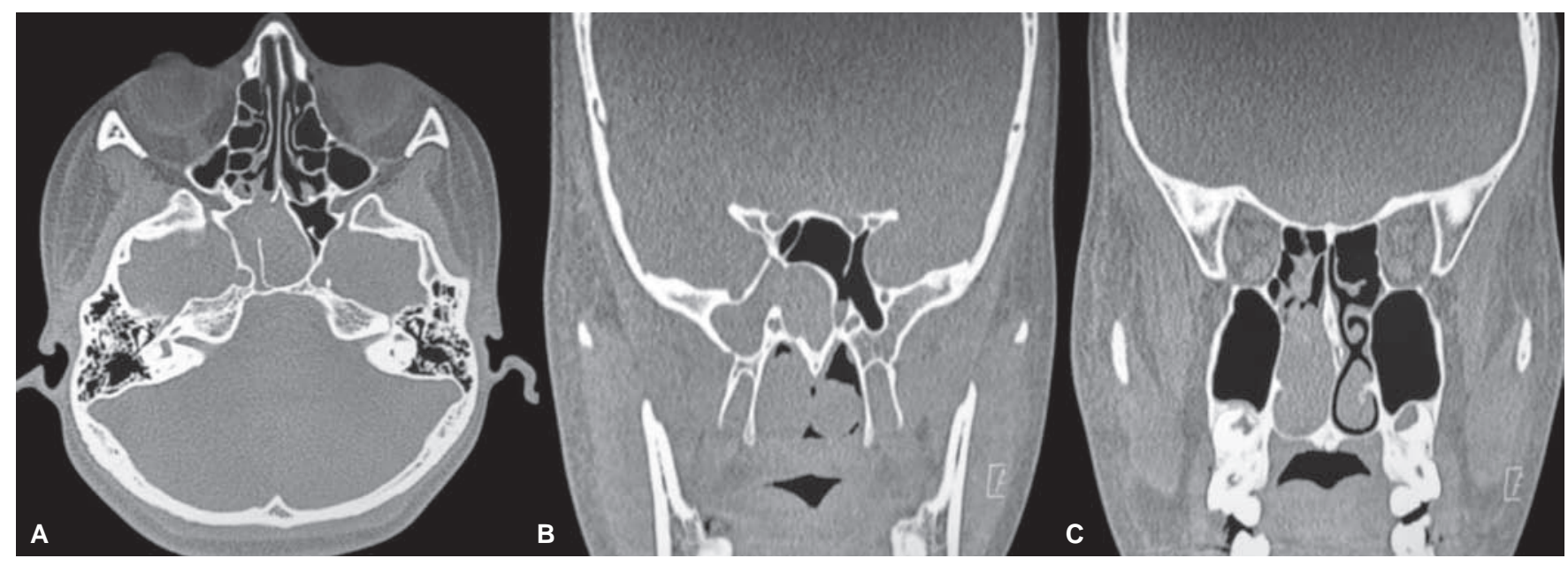

Figs 3A to C: Computed tomography scans taken from case report 2. These images showed a sphenochoanal polyp from the right sphenoid sinus
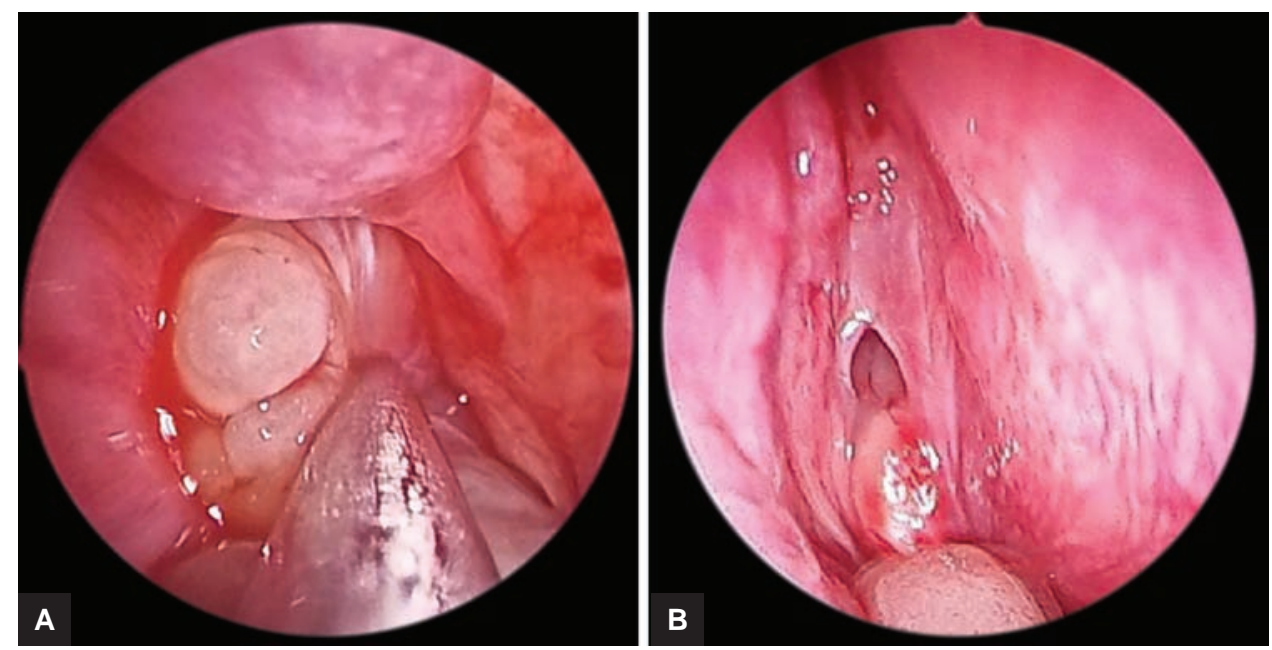

Figs 4A and B: Intraoperative pictures from case report 2 that showed the distal sphenoidal portion of sphenochoanal polyp and sphenoid ostium that was already widened by the stalk
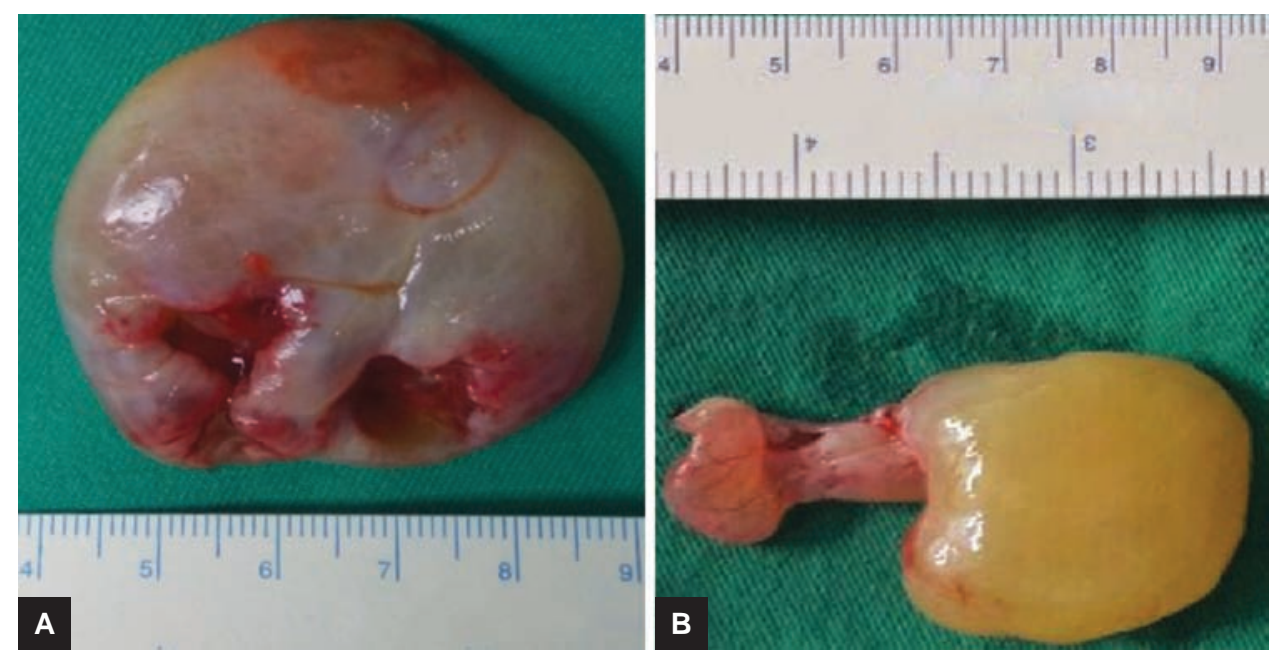

Figs 5A and B: Two large sphenochoanal polyps that were retrieved after evacuation via oral cavity, left figure from case report 1 and right figure from case report 2 
clinical characteristics. ${ }^{3}$ In the event of misidentification of SCP as ACP due to their close resemblance, a patient may be wrongly subjected to unnecessary procedure.? This may lead to incomplete removal of the polyp from the sphenoidal sinus and unjustifiable exploration of the maxillary sinus. ${ }^{7}$ Therefore, to get an accurate diagnosis and fruitful preoperative evaluation for SCP, a more comprehensive approach involving the use of the latest technology including nasoendoscopy and CT scan or MRI must be applied. 2,4,5,7 The SCP will appear as a lowly attenuated singular mass arising from the sphenoid sinus, extending to sphenoethmoidal recess and choana in the CT scan. ${ }^{5}$ Once the origin of polyp is identified with the help of CT scan like in case report 2, a surgical approach can be planned right away, even during the preoperative consultation. For less clear-cut cases like in case report 1 , where the SCP may resemble ACP in initial clinical diagnosis and the exact location of polyp cannot be specified via the CT scan, ESS proved to be not just therapeutic, but diagnostic as well. ${ }^{2,7,8}$ The ESS with transnasal approach has been regarded as ideal, as this technique permits direct visualization of the polyp's origin. ${ }^{2,8}$ In both of our cases, we approached the SCP transnasally, gradually debulking the choanal part of the SCP and then trace its pedicle up to the sphenoid sinus using microdebrider, as this method has been found to be safe and effective. ${ }^{2,7}$ Simple polypectomy using snares and forceps should be avoided as this procedure is often associated with high recurrence rates. ${ }^{3}$ Premature avulsion of the choanal portion may cause the distal portion to be retracted into the sphenoid sinus, thus encumbering the surgeon from complete excision. ${ }^{7}$ The sphenoid sinus ostium is widened for better visualization and confirmation of total excision intraoperatively while facilitating monitoring of possible recurrence via nasal endoscopy during the postoperative clinic follow-ups. ${ }^{7,8}$ The ESS is generally a safe procedure in the hands of an experienced surgeon, but with the emerging number of surgeries, the chances to be served a lawsuit following ESS, with current complication rate of 0.3 to $3.0 \%$, have increased as well. ${ }^{9}$ In order to minimize the operative risk, especially in less straightforward ESS, like in case report 1 , the usage of IGS has been reported to reduce the complication rate to 0 to $1.5 \% .^{9}$ In the management of SCP, IGS proved to be helpful in terms of increasing the confidence of surgeons, hence avoiding possible injuries to surrounding structures, such as orbit and skull base.

\section{CONCLUSION}

The SCP is indeed a rare entity that is often overlooked due to its similarity to ACP. Awareness and usage of endoscopic and imaging techniques are important to correctly identify and recognize this condition. In the event of diagnostic dilemma, ESS can both be therapeutic and diagnostic. The SCP should be approached transnasally and excised in total to minimize recurrence with the usage of microdebrider. The IGS is beneficial for nasal polyp with sphenoidal origin due to its close proximity to the vital structures in skull base and for meticulous, complete removal of the disease.

\section{REFERENCES}

1. Eloy PH, Evrard I, Bertrand B, Deelos M. Choanal polyp of sphenoidal origin. Report of two cases. Acta Otorhinolaryngol Belg 1996 Jan;50(3):183-189.

2. Crampette L, Mondain M, Rombaux P. Sphenochoanal polyp in children: diagnosis and treatment. Rhinology 1995 Mar;33(1):43-45.

3. Tosun F, Yetiser S, Akcam T, Ozkaptan Y. Sphenochoanal polyp: endoscopic surgery. Int J Pediatr Otorhinolaryngol 2001 Apr;58(1):87-90.

4. Sethi DS. Isolated sphenoid lesions: diagnosis and management. Otolaringol Head Neck Surg 1999 May;120(5):730-736.

5. Weissman JL, Tabor EK, Curtin HD. Sphenochoanal polyps: evaluation with CT and MR imaging. Radiology 1991 Jan;178(1):145-148.

6. Stammberger, H. Functional endoscopic sinus surgery. Philadelphia (PA): BC Decker; 1991.

7. Soh KB, Tan KK. Sphenochoanal polyps in Singapore: diagnosis and current management. Singapore Med J 2000 Apr;41(4):184-187.

8. Lopatin A, Bykova V, Piskunov G. Choanal polyps: one entity, one surgical approach? Rhinology 1997 Jun;35(2):79-83.

9. Farhadi M, Jalessi M, Sharifi G, Khamesi S, Bahrami E, Hammami MR, Behzadi AH. Use of image guidance in endoscopic endonasal surgeries: a 5-year experience. B-ENT 2011 Jul;7(4):277-282. 